\title{
9
}

\section{ORGANIZATIONAL AND NATIONAL ISSUES OF AN ERP IMPLEMENTATION IN A PORTUGUESE COMPANY}

\author{
Jose Esteves, Joan Pastor, João Carvalho \\ Universidad Politecnica de Catalunya, Universidad Internacional de Catalunya, Universidad \\ do Minho, Spain
}

\begin{abstract}
This paper describes a case of an Enterprise Resource Planning (ERP) implementation in a Portuguese SME. We focused on the identification of organizational factors that affected the ERP implementation project. We also analyze the ERP implementation project from a national cultural perspective using Geert Hofstede's dimensions. These dimensions are used to explain some of the attitudes and behaviours during the ERP implementation project. Our findings enforce that some of the problems in ERP implementation projects are not of technological nature but may be attributed to organizational factors while some issues are related to national culture.
\end{abstract}

Keywords: Enterprise Resource Planning, ERP implementation, grounded theory, organizational culture.

\section{INTRODUCTION}

In recent years, it has been demonstrated that Small and Medium sized Enterprises (SMEs) strongly contribute to national economies. SMEs constitute around 95 percent of enterprises and account for up to 70 percent of employment in most countries around the world. The adoption of ERP systems is now reaching SMEs, bringing up problems in ERP implementation projects which are specific to this type of companies. This paper describes the results of a case study carried out in a Portuguese SME that implemented the SAP R/3 system in 1998 by following a big-bang 
implementation approach. The big-bang approach is characterized by the installation and go live of all implemented ERP modules at the same time. The interpretive perspective adopted in our research reflects our aim for understanding the phenomenon of ERP implementation in a SME within the organizational and national contexts where it occurs. The paper is structured as follows. First we present the research methodology; in this study we combined the case study method with the grounded theory method. Next, we describe the case study background followed by a presentation of its findings. Hofstede's (1991) dimensions of national culture are then related to the case study. Finally, the conclusions and the implications for further research are outlined.

\section{RESEARCH METHODOLOGY}

As we believe that the understanding of ERP implementation cannot be achieved without considering the organizational context where it occurs, the chosen research method was the in-depth case study method (Yin, 1994). In order to identify the organizational factors that affect an ERP implementation in SMEs we opted for an interpretive research approach. Interpretive research does not predefine dependent or independent variables and it attempts to explain phenomena through the meanings that people assign to them (Orlikowski and Baroudi, 1991).

We started the case study by defining a plan to collect data. We first analyzed documentation created during the SAP implementation project that was provided to us by the project manager. The documentation helped to understand the project background and to prepare the questions for the interviews. The main technique chosen for data collection was semistructured interviews. Following the contact with key informants in the company, interview schedules were agreed upon. Interviews were tape recorded and transcribed to ensure accuracy of written data, and to minimize researcher's bias. Initially, three interviews were made with the project manager, another member of the project team and a key-user. Then, we interviewed the remainder members of the project team. Data from interviews was triangulated with the documentation so far accumulated. In order to build theory from this case study, we adopted the Grounded Theory (GT) method.

GT is a general method developed by Glaser and Strauss (1967) for building theories that are grounded in data systematically gathered and analyzed (Glaser and Strauss, 1967). Strauss and Corbin (1990: p.23) explain that by using GT "a theory is inductively derived from the study of the phenomenon it represents. That is, it is discovered, developed, and 
provisionally verified through systematic data collection, analysis, and theory stand in reciprocal relationship with each other. One does not begin with a theory, and then prove it. Rather, one begins with an area of study and what is relevant to that area is allowed to emerge". The coding process of all interviews and documentation allowed major themes/categories to emerge. Then, we used the paradigm model proposed by Strauss and Corbin (1990) to relate these categories. Briefly, the paradigm model encompasses the following elements: causal conditions, the phenomenon, the context, the intervening conditions, strategies and actions, and finally the consequences. Finally, in order to analyze the national culture perspective, we used Hofstede's (1991) dimensions. The paradigm model context was thus extended with an additional category: national context. The national perspective is explained in Section 5.

\section{CASE STUDY BACKGROUND}

PhotoPics S.A. (fictitious name) is the Portuguese subsidiary of the PhotoPics multinational company. The PhotoPics Group was created in Germany around 1849 to develop lenses and microscopes. The company started in Portugal in 1973 and the Portuguese unit is now the main one of the PhotoPics Group worldwide. It has a total of 660 employees, most of them wage earners with approximately 100 salaried employers. Table 1 describes the different actions taken to improve information technology infrastructure in PhotoPics.

Table 1. Information technology evolution in PhotoPics

\begin{tabular}{ll}
\hline Period & Actions \\
\hline Until 1997 & - Only dumb terminals connected through a dedicated infrastructure. \\
& - Comet Top software package to support logistics, production planning, \\
& payroll and accounting. \\
& - Only the administrator and the financial director used PCs. \\
& - No internet connection. \\
\hline Beginning 1997 & - Analysis of Comet Top upgrade or adoption of an ERP system due to \\
& Y2K problem and Euro conversion. \\
\hline First quarter 1997 & - Decision to adopt SAP system. \\
\hline 1997 & - Implementation of a computer network. \\
& - Upgrade of Comet Top to a Unix version until SAP go live. \\
& - Training of users on using PCs (average age of users was 40 years old). \\
\hline
\end{tabular}




\subsection{ERP Implementation Phases}

PhotoPics followed a typical ERP implementation lifecycle with a bigbang approach. The project goal was the implementation of SAP R/3 system, version $3.1 \mathrm{H}$ with the main SAP modules. The number of expected endusers was 30 to 40 . At the beginning of the project, it was estimated that no be-spoke development would be made and that only enhancements to forms and reports would be tailored. Table 2 describes the implementation phases.

Table 2. ERP implementation phases in PhotoPics

\begin{tabular}{ll}
\hline Phase & Description \\
\hline Planning & This was the basis for the entire project. The goal of this phase was to detail the \\
project definition and its functional needs. The project structure was defined. \\
This phase was arduous due to three main aspects: the definition of all \\
processes that attempted to be implemented in the new system, contact with all \\
the process stakeholders, and the difficulty to obtain information. \\
The goal of this phase was to produce the technical specification of how to \\
implement the chosen solution and the beginning of the parameterization and \\
the preparation of a prototype that allowed the demonstration of the system \\
wesign \\
working for each planned situation. This phase was felt as fundamental for the \\
system comprehension since the internal project team took its first contact with \\
the SAP system. \\
The goal of this phase was to obtain the configuration of the SAP system \\
according to the design, the development of some complementary programs \\
that served as interfaces to SAP, and the creation of training manuals and final \\
tests. \\
The goal of this phase was to put the new system at work. The go-live phase \\
was started a month behind schedule given some changes in the scope of the \\
project. The expressions "the company will stop" or "it will not work" were in \\
the mind of everyone, but everything worked perfectly. At the end of this phase \\
an analysis of the general difficulties of the SAP implementation project was \\
made.
\end{tabular}

\section{ERP IMPLEMENTATION PROJECTS IN SMES: ORGANIZATIONAL PERSPECTIVE}

In this section we describe the paradigm model (see Figure 1) that we developed. The paradigm model includes both, the technological and organizational perspectives. 


\subsection{Phenomenon}

Strauss and Corbin (1990: p.101) stated that phenomena are "the central ideas in data represented as concepts". According to their account, the purpose behind naming phenomena is to enable researchers to group similar events, happenings, and objects under a common heading or classification. The phenomenon in the paradigm model is represented by the central category (sometimes called core category), which represents the main theme of the research. The phenomenon addressed in this study is the implementation of an ERP system in a SME. ERP implementation at PhotoPics was considered to be a successful one.

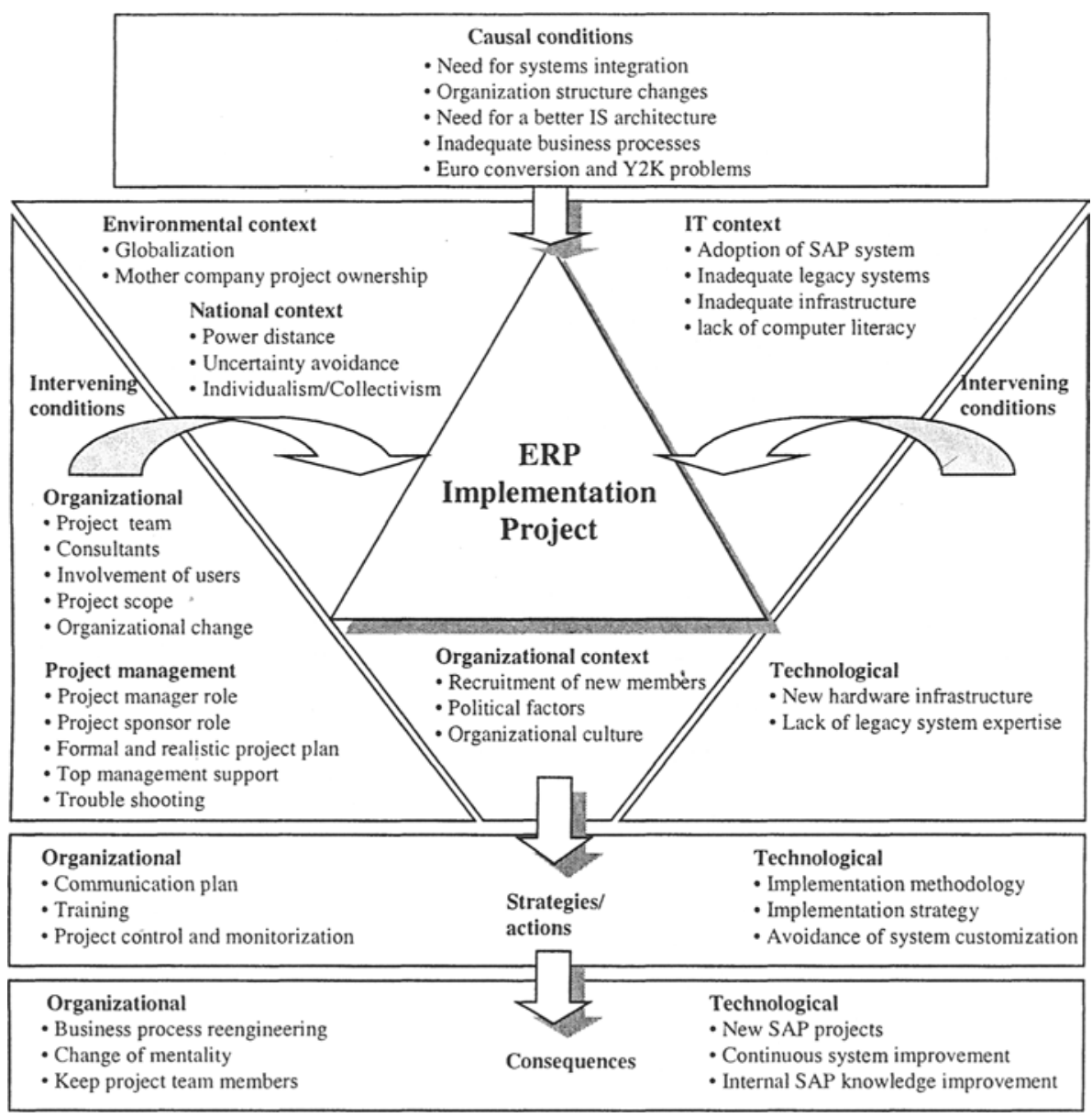

Figure 1. A model for ERP implementation projects in SME 


\subsection{Causal Conditions}

The circumstances that led to the ERP implementation at PhotoPics were: the need for systems integration worldwide at PhotoPics Group; the need for a better IS architecture at PhotoPics SA; most of the business processes were inadequate and they needed a strong reengineering effort; and the mentioned Euro conversion and Y2K problems. In the first quarter of 1997 the company selected the SAP R/3 system. The main reason was that this software seemed to be the best answer to the company needs. The fact that SAP is a German software certainly transmitted security to a German company and also contributed to the decision.

\subsection{Context}

\subsubsection{Environmental Context}

By the time ERP implementation at PhotoPics took place, many other companies, including SMEs, were leaving their legacy systems and migrating to ERP systems. Again, problems related with the Y2K and the unavoidable changes in IT systems related to the forthcoming adoption of a single currency by most of the EU countries were viewed as strong justifications for carrying out deep changes on IT systems. The parent company decided to integrate the information of all its subsidiary companies worldwide. Therefore, the company decided to unify information and to have a better control of its subsidiary organizations such as the Portuguese PhotoPics. The parent company decided to adopt SAP R/3 as the common ERP system worldwide. The parent company kept the ownership of the project implementation, but each local organization had total freedom in the implementation process.

\subsubsection{Organizational Context}

Before the SAP implementation project started, the administration decided to recruit new members, with academic education and proved skills and experience in their field. At that moment, PhotoPics had a conservative organizational culture with power and influences concentrated in just a few members with long careers within the organization. Almost all the senior staff had no academic education and their whole career had been made within the organization. The channels of power and communication were quite complex. Most of the business processes were complex and inefficient. Technology and innovation were associated with costs and troubles. Political 
factors had a strong impact on this project. The interests of stakeholders within the organization were challenged as senior and middle level managers lost power and influence. Many employees feared losing their jobs or that such an expensive project could originate an organization rupture. They also feared the expected increase on their expected efficiency with performance control measures. Some managers intentionally concealed information about their intentions with the SAP system to team members, some other managers disseminated false information in attempts to discredit the SAP project, while others avoided participating in the project. Top executives faced an internal conflict as they were being forced to carry out changes that they felt could be against their interest. Conflicts among senior managers arose as they tried to dominate and impose their view in the SAP project, since most of them were aware that the results of the SAP project could influence their future career within the organization.

\subsection{Intervening Conditions}

\subsubsection{Organizational}

Internal project team - The implementation project structure is represented in Figure 2. Most of the internal team members were new to the organization. The project team included young university graduates that recently completed their studies. Although these people had no organizational nor SAP knowledge, they had very good skills and they were very motivated to learn. The purpose of hiring these people was to prepare them for performing to work in the organization and to develop internal knowledge in SAP. This also minimized the risk of expertise turnover.

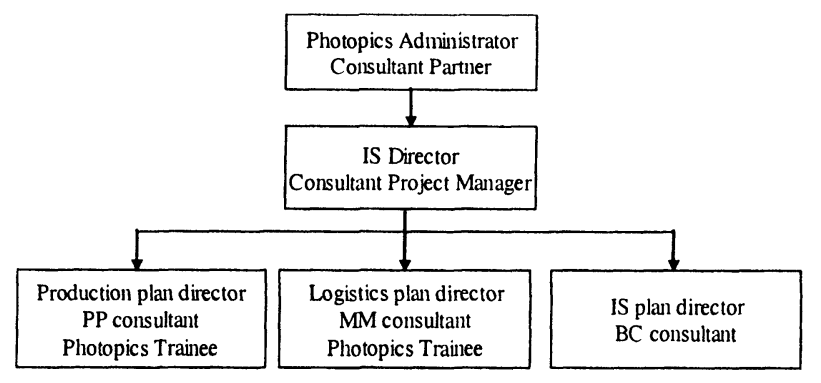

Figure 2. The ERP implementation project structure

Since the team members, which were hired specially for the SAP implementation, did not know the company well, they could carry out the 
analysis process from a more neutral position and work on the improvement of business processes without bias. The project team included key-users and chief department directors. These directors were assigned the responsibility of the SAP modules corresponding to their own departments. According to the project manager, the responsibility for implementing each module was given to each current domain manager, but logistics and IS directors were new within the organization. Currently, they admit as weaknesses their lack of knowledge about the business and organizational culture and politics.

External consultants - Consultants played an important role in the whole project. There was a careful selection of consultants according to their skills. The consultant selection made by PhotoPics was due to: SAP recommendation, the geographic location, and because the PhotoPics financial auditors knew the consulting company. However, at the beginning of the project, some of these consultants abandoned the project as they moved to other consulting companies. Due to the consulting company lack of experts at that moment, most of the newly allocated consultants were junior consultants with lack of knowledge in SAP projects and the PhotoPics business. The positive side of these junior consultants was their motivation and dedication to the project. As soon as the team members started getting SAP knowledge, they decided which consultants should remain and which new consultants should be hired. There was a lot of turnover in relation to consultants with some of them remaining only part-time in the project. Since the project manager felt quite insecure with the consulting company, he decided to hire other consultants from a second consulting company to cooperate in specific activities of the project. The role of these 'impartial' consultants was crucial to improve the quality of the project and to help the project team to make decisions in aspects about which it lacked knowledge.

User involvement - Users were involved in the project even though some claimed the contrary. Due to their lack of business knowledge, team members interviewed users in the analysis process, and managers approved the processes procedures. In most cases users themselves did the process analysis for their specific processes. Key-users were particularly relevant in this task and during tests. Their involvement also helped them to adapt to the new system. They brought in operational knowledge and they served as enablers of change in their part of the organization. Members of the project team reported that the major problem when involving users is that each user thinks his/her opinion is more important and valuable than that of others (see political factors in organizational context section).

Organizational change - Probably, due to the lack of experience in change management from the project manager and the consultants, there was not a formal organizational change plan. The organizational change affected all levels and aspects such as organizational structure and culture. Probably 
the most important change was the creation of the logistics department that took over logistics functions carried out before by the financial department. Most interviewees mentioned that organizational change procedures should exist, since several job descriptions were changed and/or eliminated and in some cases training needs could have been predicted. In most cases that aspect was analyzed a posteriori. Top management started, facilitated and supported the organizational changes and implied decision-making tasks during the project. Another aspect was that the changes were visible only within a one year span after the system go-live. The organization needed one year to adapt, as well as to understand the new organizational model.

Project scope - The project scope definition was quite limited. It focused mainly on the modules and the functions to be implemented. The lack of project strategic vision was a weakness. Since the SAP system was imposed, the project manager and top management did not carry out a strategic analysis of the implementation, especially in what concerns longterm impact. Goals were not properly defined at the beginning. The single goal to be expressed was that SAP implementation would be carried out within a determined period.

\subsubsection{Project Management}

Project manager - The project manager was recruited specifically for the SAP implementation project. He made the connection between the implementers (internal team members and external consultants) and top management. He now admits that someone with experience in SAP would have performed better the job, especially at the early stages of the project. The project manager stresses that skills to manage conflicts and people are crucial in a project of this kind. He says that a SAP project is a task of managing willingness, expectations and conflicts rather than an IT implementation project. As PhotoPics is owned by a German company, mastering the German language was an important competence for the project manager although that was not considered at selection time. This was fundamental to communicate and share knowledge with the parent company and it contributed to solve many problems and to improve collaboration.

Top management support - Top management support and commitment were critical to achieve success in the project. Top managers were always available to discuss doubts and trouble-shooting and to make prompt decision-making in order to avoid bottlenecks. This commitment was vital for disseminating the project to the whole organization and especially for dealing with SAP opponents. It was the organization CEO who played the role of project sponsor. According to most interviewees he was the person that drove forward the SAP project. 
Trouble shooting - Several problems appeared along the SAP project mainly due to project team inexperience and to the political factors described above. Two main issues affected the SAP project: lack of knowledge about the legacy systems and the difficulties with data conversion. In what concerns the legacy system, there was lack of documentation and there were no experts in the system. Most of the information was obtained via the parent company. The data dealt with by the legacy system was not enough for the new system, or it was not structured according to the new business model. Therefore, there was the need to create several conversion programs and for some manual data insertion. With the SAP knowledge acquired meanwhile, the project team admits there were better data conversion solutions that those adapted in the project.

\subsection{Action/ Interaction Strategies}

Communication plan - The communication plan was divided in two types: communication inwards (between team members and with top management) and communication outwards (with the rest of the employees). Inwards communication worked very well. A special room was allocated to team members that facilitated knowledge sharing and cooperative work. In what concerns outwards communication, the project team regularly presented newsletters, invitations to participate in promotion events, and an intranet was implemented. However, the interest of employees was minimal. As the project manager and some team members mentioned, employees were more worried on how much money was being spent.

Training - The consultants proposed a training plan that was accomplished. However, team members complained about their training: training was made at the end of the design phase instead of the end of the planning or at least the beginning of analysis phase; training was very basic and focused in the potential of SAP, and trainers had little training experience. Therefore, team members developed their own skills based in self-study during the project. The first step in end-users training was to define a training plan that included basic training in computers use because almost all the users were computer illiterate. The training started early for end-users. This helped to keep them in contact with the system and to start the process of adaptation to SAP. The project manager noted that starting training too early might be a problem because some users tend to forget what they learn, which implies continuous refreshment training courses. On the other hand, late training will bring problems to the go-live phase and extra effort for team members. He also recommends to use a SAP parameterization as close as possible to the final one since this provides a better and quick adaptation to what users will have to deal with after go-live 
phase. One thing that failed in the training plan was new or changed business domain training. The lack of such training affected organizational change. The project manager admits that an ERP training plan must be a mix of technical and organizational training.

Project monitoring and control - Project monitoring and control mainly consisted on verifying the accomplishment of the project plan and schedule. With no goals defined at the beginning and with the lack of experience in project management, there were no project metrics being used. However, project meetings were used to continuously monitor and control the project. There were weekly meetings with project team and monthly meetings with top managers. At the end of each phase there was a meeting with all managers. The estimated duration of the project plan was not achieved but all the scheduled tasks were performed.

\subsection{Consequences}

Business process reengineering (BPR) - Although BPR started in the gap analysis, its effective change occurred in the go-live phase. Parallel to the implementation of the SAP system, managers started changing the business processes while explaining it to the organization. The project team admits that this should have been done before the implementation since some processes were totally obsolete or inadequate. BPR is not finished yet. Nowadays, during the post-implementation period and using the knowledge that the project team has at the present, they are always improving processes by extending processes functionality through the current discovery of functionality in the SAP system. The SAP system brought in new business processes and helped in the reengineering of the old ones. As the project manager mentioned, PhotoPics is a manufacturing organization with no unique processes. Therefore, they would not loose competitiveness by adopting SAP best practices. On the contrary, the adoption of practices that SAP provides helped to reengineer the existent business processes and simplified implementation and future maintenance efforts.

Change of mentality - One of the most interesting consequences of this SAP implementation was the induced change of mentality. From the beginning, most of the users disagreed with the implementation of the system but their attitude reversed progressively after the go-live phase. Nowadays, they recognize that SAP is useful in having helped to improve the business and they think that more business analysis should still be done, with managers demanding more people to improve the work. The main reasons for the changeover were: top management commitment, the continuous training and the support of team members. Some users were moved from their old functions while others had intensive training. Now, 
only a few employees are still not using the system. Some of them were middle managers that, due to their age and education, had difficulties to adapt to the new system. The solution was to train some subordinates of these middle managers that would then help them in the tasks that demand the use of SAP. Nowadays, users are no longer afraid of changes. They are aware that the system is something about continuous improvement process.

Keep team members - In relation to the project team, the young graduates were incorporated into the organization with substantial increase of salaries. This helped to keep and improve the SAP knowledge in the organization and avoid dependency on consultants. A continuous learning process was developed with continuous training in SAP in order to improve SAP internal knowledge.

\section{ANALYSIS FROM A NATIONAL PERSPECTIVE}

After we created the ERP implementation model, we decided to try understanding better the organizational context of PhotoPics. Based on Hofstede's (1991) study of national cultures and organizations, we analyzed the findings of PhotoPics case study from three dimensions: power distance, uncertainty avoidance and individualism-collectivism. Table 3 describes the dimensions and the values for Portugal.

Table 3. Cultural dimensions suggested by Hofstede (1991) with score and mean for Portugal

\begin{tabular}{llll}
\hline Dimension & Description & Score & Mean \\
\hline Power & $\begin{array}{l}\text { Power distance is the degree to which members of a society } \\
\text { accept as legitimate that power in institutions and }\end{array}$ & 51 \\
distance & $\begin{array}{l}\text { organizations is unequally distributed (Hofstede 1991). } \\
\text { Higher power distance scores indicate an order of inequalities, } \\
\text { special privileges for those of higher status, superiors consider } \\
\text { subordinates as a different kind of person. }\end{array}$ & \\
Uncertainty & $\begin{array}{l}\text { Uncertainty avoidance is the degree to which members of a 104 } \\
\text { avoidance }\end{array}$ & $\begin{array}{l}\text { society feel uncomfortable with uncertainty and ambiguity, } \\
\text { which leads them to support beliefs that promise certainty and }\end{array}$ \\
& $\begin{array}{l}\text { to maintain institutions that protect conformity (Hofstede } \\
\text { Individualism }\end{array}$ & \\
/ Collectivism & $\begin{array}{l}\text { Hofstede (1991) proposes a single dimensional structure 27 } \\
\text { called individualism-collectivism, where those cultures that } \\
\text { emphasize the autonomy of the person are grouped under }\end{array}$ & \\
& individualism, while those cultures whose most important \\
& $\begin{array}{l}\text { values place emphasis on the dependency of the individual } \\
\text { with respect to groups are clustered under collectivism. }\end{array}$ & \\
\hline
\end{tabular}


Next, we relate and discuss each dimension with the PhotoPics SAP implementation project.

\subsection{Power Distance}

The score of Portugal is 63 (mean 51), which means that Portugal has a high power distance score. The political and culture issues emerged as important factors in the organizational environment in our case study of PhotoPics (see context section above). In this case, we emphasize the issue of losing organizational power and influence. Most of these managers had a large career within the organization and they controlled their departments with authority like 'feuds' as one of the interviewees mentioned. Each department director filtered the information for the organization owner and the one shared among managers. Conflicts among senior managers arose as they tried to dominate and impose their view in the ERP project, since most of them were aware that the results of the ERP project could influence their future career. Since managers were not happy with the project, they passed the negative image to their subordinates, which caused more general disagreement with the project.

\subsection{Uncertainty Avoidance}

According to Hofstede (1991), the Portuguese society has a high level of uncertainty avoidance, scored 104 (mean 64). In the case of PhotoPics the uncertainty was very high during the whole project. Uncertainty was mainly due to the people being afraid of losing power (top and middle management) and lower levels, where people were afraid of losing their jobs because of organization bankruptcy or the decision to reduce the number of employees. This uncertainty made some managers express their disagreement during the project and some of them difficulting the process by omitting some information, lack of participation in some implementation tasks, no appearance to some important meetings, and decision-making delays in order to delay the whole ERP project.

\subsection{Individualism-Collectivism}

According to Hofstede (1991), the Portuguese society is a collectivist society. PhotoPics workers had been doing their tasks in the same way during many years and they were not happy in changing the way of working. One of the reasons for this disappointment was their age, most of the employees were over 50 with more than 20 years working at PhotoPics. Some of them were also expecting retirement (the legal age is 65 years). The 
work relationships were in some sense like being part of a family. Hofstede (1991) states for this case that the relationship between owner and employee is viewed in a moral perspective, and is like a familiar relationship were both have obligations: protection in exchange of loyalty. Most of the employees of PhotoPics doubted about the real intentions from the new top management with the introduction of this new expensive SAP system. There was a notorious lost of trust on their managers what made that most employees started showing indifference to the SAP project.

\section{CONCLUSIONS AND IMPLICATIONS FOR FURTHER RESEARCH}

We think that the main conclusion of this study is the evidence that organizational, cultural and national issues have a strong impact in ERP implementation projects. In the particular case addressed in this study if those organizational and cultural issues had been taken into account during the planning phase, it is very likely that some problems would have been avoided or mitigated during the ERP implementation project.

Skok and Doringer (2000) pointed out the need to study the relevance of macro and micro-level cultural issues in the successful operation of ERP systems focusing on the function versus process view of ERPs. Our findings suggest that the relevance of these studies goes beyond the use of ERPs. The analysis of Hofstede's dimensions in our case study suggests that these dimensions could be helpful in addressing some ERP project issues and, consequently, to define a strategy for ERP implementation. For instance, one of the main issues in ERP implementation projects is the project duration which is often used as one of the ERP project success evaluation indicators. Kale (2000) evidences the impact of cultural readiness on ERP project duration, which supports our preliminary findings. These findings can also help in defining the determinants of Critical Success Factors (CSFs) for ERP projects (Esteves and Pastor, 2000). Research on CSFs identification does not provide the reason why some CSFs are more relevant in some organizations than others. In this study we analyzed Hofstede's dimensions one by one. Future research should also consider the influence of associations of dimensions like power distance/uncertainty avoidance or uncertainty avoidance/individualism effects. The literature on IS evidences a lack of research on corporate and national culture. The influence of culture has mainly been focused in the IS solution and how it fits with the organizational culture, or the cultural changes. Another aspect to consider is whether ERP implementations significantly differ from the implementation of other types of systems in the past or currently (e.g., datawarehousing, 
CRM, SCM). This research path can bring up conclusions regarding the specificity of ERP implementation projects or the identification of general trends in IS implementation.

\section{REFERENCES}

Esteves, J., and Pastor, J. "Towards the Unification of Critical Success Factors for ERP implementations", 10th Annual BIT conference, Manchester, UK, 2000.

Glaser, B., Strauss, A. The Discovery of Grounded Theory: Strategies for Qualitative Research, New York: Aldine de Gruyter, 1967.

Hofstede, G. Cultures and Organizations: Software of the Mind, New York: McGraw-Hill, 1991.

Kale, V. Implementing SAP R/3: The Guide for Business and Technology Managers, Indiana, USA: SAMS publications, 2000.

Orlikowski, W., and Baroudi, J. "Studying Information Technology in Organizations: Research Approaches and Assumptions”, Information Systems Research, (2:1), 1991, pp. $1-28$.

Skok, W., and Doringer, H. "Potential Impact of Cultural Differences on Enterprise Resource Planning (ERP) Projects", Electronic Journal on Information Systems in Developing Countries, (7:5), 2000, pp. 1-8.

Strauss, A, and Corbin, J. Basics of Qualitative Research: Grounded Theory Procedures and Techniques, Newbury Park, CA: Sage Publications, 1990.

Yin, R. Case Study Research: Design and Methods, Thousands Oaks, CA: Sage Publications, 1994.

\section{About the Authors}

José Esteves is a doctoral student in the IS doctoral program of the Technical University of Catalonia, Barcelona, Spain. He is computer engineer, has a master in IS, and a diploma in business administration. His research interests include ERP systems, knowledge management and qualitative research. He can be reached by e-mail at jesteves@lsi.upc.es.

Joan Pastor is an associate professor and Dean of the IT school of Universidad Internacional de Catalunya, Barcelona, Spain. He holds a degree in computer science and a Phd in software. He is also leading the "Twist" group on IS qualitative research, addressing topics like ERP procurement and implementation. He can be reached by e-mail at jap@unica.edu.

João Carvalho is an associate professor and Head of the Information Systems Department at University of Minho, Portugal. His research interests include the fundamentals of information systems, knowledge management and research methodology. He can be reached by e-mail at jac@dsi.uminho.pt. 